\title{
Quantum Zeno effects across a parity-time symmetry breaking transition in atomic momentum space
}

\author{
Tao Chen ${ }^{1}$, Wei Gou $\mathbb{D}^{1}$, Dizhou Xie ${ }^{1}$, Teng Xiao $\mathbb{D i}^{1}$, Wei Yi $\mathbb{D}^{2,3}$, Jun Jing ${ }^{4 凶}$ and Bo Yan ${ }^{1,5,6 凶}$
}

We experimentally study quantum Zeno effects in a parity-time (PT) symmetric cold atom gas periodically coupled to a reservoir. Based on the state-of-the-art control of inter-site couplings of atoms in a momentum lattice, we implement a synthetic two-level system with passive PT symmetry over two lattice sites, where an effective dissipation is introduced through repeated couplings to the rest of the lattice. Quantum Zeno (anti-Zeno) effects manifest in our experiment as the overall dissipation of the two-level system becoming suppressed (enhanced) with increasing coupling intensity or frequency. We demonstrate that quantum Zeno regimes exist in the broken PT symmetry phase, and are bounded by exceptional points separating the PT symmetric and PT broken phases, as well as by a discrete set of critical coupling frequencies. Our experiment establishes the connection between PTsymmetry-breaking transitions and quantum Zeno effects, and is extendable to higher dimensions or to interacting regimes, thanks to the flexible control with atoms in a momentum lattice.

npj Quantum Information (2021)7:78; https://doi.org/10.1038/s41534-021-00417-y

\section{INTRODUCTION}

Coherent evolution of a quantum system can be frozen when frequently interrupted by measurements or perturbations. Such a phenomenon, famed as the quantum Zeno effect, has been experimentally observed in various physical systems ${ }^{1-5}$, and has found widespread utilities in quantum information ${ }^{6-16}$ and quantum simulation ${ }^{17-21}$. In a complementary fashion, with an appropriate repetition rate of measurements, the evolution of the system can also be accelerated under what is known as the antiZeno effect ${ }^{22}$. Intriguingly, both quantum Zeno and anti-Zeno effects are alternatively accessible through continuous strong couplings or fast unitary kicks ${ }^{3,17,23}$ that couple a system to an auxiliary Hilbert space. With the auxiliary Hilbert space playing the role of environment, these processes give rise to dissipative system-reservoir couplings, under which the time evolution of the system is effectively driven by a non-Hermitian Hamiltonian. Since a dissipative system under non-unitary evolution driven by a nonHermitian Hamiltonian is not norm-preserving and necessarily decays, the quantum (anti-)Zeno effects therein manifest as the suppression (enhancement) of decay.

Although evidence of quantum Zeno effects have been theoretically demonstrated and experimentally observed in nonHermitian settings ${ }^{24,25}$, surprisingly little is discussed on its interplay with parity-time (PT) symmetry, despite the latter being a ubiquitous property of non-Hermitian systems while holding great promise for future applications ${ }^{26-28}$. A PT symmetric, nonHermitian system possesses two distinct phases: the parity-time symmetric (PTS) phase, with entirely real eigenenergy spectrum; and the parity-time broken (PTB) phase, where eigenenergies are complex in general. The two phases are separated by exceptional points, with coalescing eigenstates and eigenenergies. While quantum Zeno effects naturally emerge in the deep PTB regime that can be mapped to an open system possessing continuous and strong coupling with a dissipative reservoir ${ }^{29}$, the fate of quantum Zeno (anti-Zeno) effects is less well known in the PTS regime or near exceptional points, both of which typically occur at much smaller dissipation strengths ${ }^{29-31}$. A very recent theoretical study shows that exceptional points of a PT symmetric Hamiltonian also mark the boundary between quantum Zeno and anti-Zeno regimes ${ }^{32}$, suggesting a deep connection between the two previously independent fields of study. Here we experimentally confirm such a connection in a PT symmetric, synthetic two-level system, embedded in a momentum lattice of cold atoms.

\section{RESULTS}

PT symmetry breaking transitions in a dissipative two-level system

We focus on a two-level system under time-periodic dissipation ${ }^{32}$, as illustrated in Fig. 1a. The time-dependent Hamiltonian is

$H / \hbar=-\frac{i \gamma(\tau)}{2} \mathbb{I}+t \sigma_{\mathrm{x}}+\frac{i \gamma(\tau)}{2} \sigma_{\mathrm{z}}$,

where $\mathbb{I}$ and $\sigma_{x, z}$ are the identity and Pauli matrices respectively, $\tau$ is the evolution time, and $t$ is the inter-state coupling rate. The time-periodic dissipation rate $\gamma$ is given by

$$
\gamma(\tau)=\left\{\begin{array}{ll}
\gamma_{0} & j T \leq \tau<j T+\tau_{0} \\
0 & j T+\tau_{0} \leq \tau<(j+1) T
\end{array},\right.
$$

where $j \in \mathbb{Z}$, the modulation period $T=2 \pi / \Omega$ with $\Omega$ the modulation frequency, $\gamma_{0}$ characterizes the modulation intensity, and $\tau_{0}$ is the duty time interval with nonzero $\gamma$ in each cycle; see Fig. $1 \mathrm{~b}$.

Hamiltonian (1) is passive PT symmetric, in that it is purely dissipative, but is directly related to the standard PT symmetric

\footnotetext{
${ }^{1}$ Interdisciplinary Center of Quantum Information, State Key Laboratory of Modern Optical Instrumentation, and Zhejiang Province Key Laboratory of Quantum Technology and Device of Physics Department, Zhejiang University, Hangzhou, China. ${ }^{2}$ CAS Key Laboratory of Quantum Information, University of Science and Technology of China, Hefei, China. ${ }^{3}$ CAS Center For Excellence in Quantum Information and Quantum Physics, Hefei, China. ${ }^{4}$ Department of Physics, Zhejiang University, Hangzhou, China. ${ }^{5}$ Collaborative Innovation Centre of Advanced Microstructures, Nanjing University, Nanjing, China. ${ }^{6}$ Key Laboratory of Quantum Optics, Chinese Academy of Sciences, Shanghai, China. ${ }^{凶}$ email: jingjun@zju. edu.cn; yangbohang@zju.edu.cn
} 
a

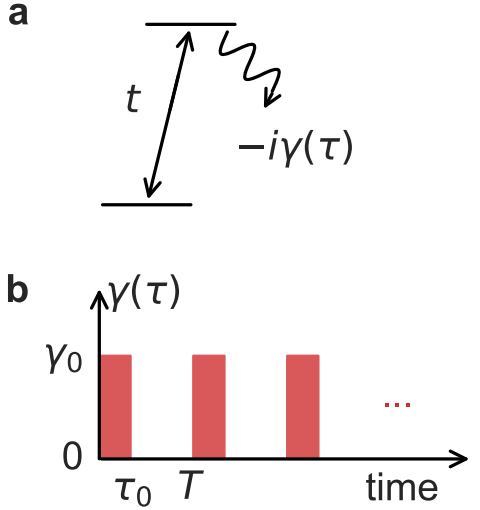

C

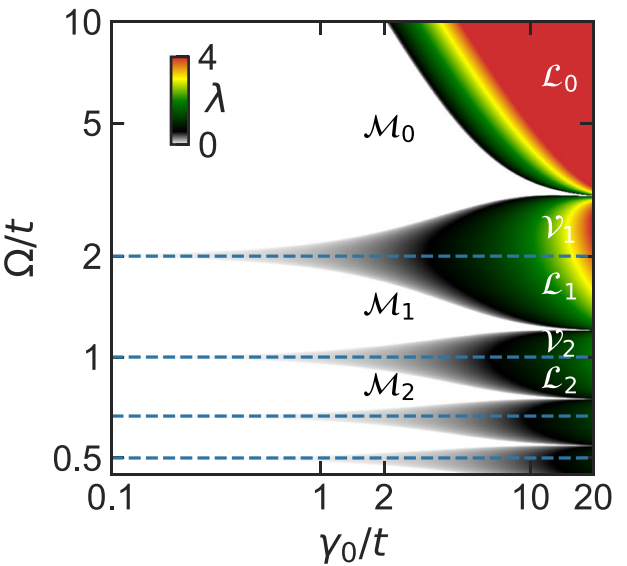

Fig. 1 PT phase diagram. a Schematic illustration of the dissipative two-level system under Hamiltonian (1). b The loss rate $\gamma(\tau)$ in panel (a) is time-periodic with a square-wave modulation. The modulation period $T=2 \pi / \Omega\left(\Omega\right.$ is the modulation frequency), and $\gamma(\tau)=\gamma_{0}$ during the duty time interval $\left[0, \tau_{0}\right]$. c Theoretical phase diagram in the $\Omega-\gamma_{0}$ plane. Color contour shows the dimensionless parameter $\lambda$ (see main text for definition). Here we set $\tau_{0} t /(2 \pi)=0.1$. Regions with vanishing $\lambda$ correspond to the PTS phase $\left(\mathcal{M}_{j}\right)$, while the colored regions $\left(\mathcal{L}_{j}\right.$ and $\left.\mathcal{V}_{j}\right)$ correspond to the PTB phase. The horizontal dashed lines indicate critical coupling frequencies separating the $\mathcal{L}_{j}$ (quantum Zeno) and $\mathcal{V}_{j}$ (quantum anti-Zeno) regimes.

a

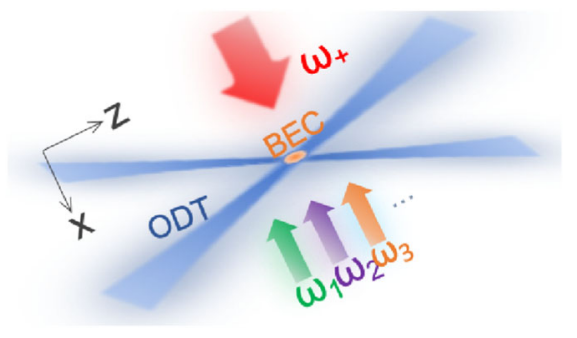

C

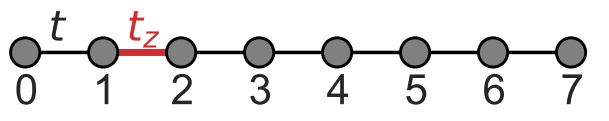

b

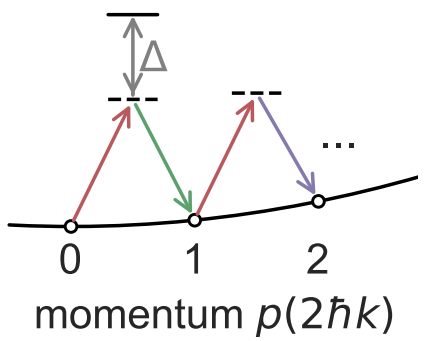

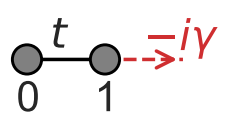

Fig. 2 Realization of a dissipative two-level system in momentum-state lattice. a Schematic of the experimental setup. A Bose-Einstein condensate interacts with a pair of counter-propagating Bragg lasers in an optical dipole trap. The beam propagating along the $-x$ direction contains multiple frequency components $\left(\omega_{j}\right.$ with $\left.j=1,2, \ldots\right)$ whose intensities and frequencies can be precisely controlled (see Supplemental Information). b Each Bragg laser pair $\left\{\omega_{+}, \omega_{n}\right\}$ triggers a resonant two-photon Bragg transition, coupling two neighboring momentum states $\left|p_{n-1}\right\rangle \leftrightarrow\left|p_{n}\right\rangle$ along the momentum lattice. c The resulting 8-site momentum lattice is mapped into a dissipative two-level system with tunable loss rate $\gamma(\tau)$, by treating the sites $\{|0\rangle,|1\rangle\}$ as the system, and $|n \geq 2\rangle$ as a reservoir.

Hamiltonian $\hbar \epsilon_{ \pm}$with balanced gain and loss. Explicitly, $\mathcal{P} \mathcal{T} H_{P T} \mathcal{P} \mathcal{T}^{-1}=H_{P T}$, with the PT symmetry operator $\mathcal{P} \mathcal{T}=\sigma_{x} \mathcal{K}$ where $\mathcal{K}$ is complex conjugation. Since PT symmetry of $H$ is determined by the imaginary parts of the quasienergies $\hbar \epsilon_{ \pm}$of the corresponding Floquet Hamiltonian 31,32 , we adopt a dimensionless parameter $\lambda=\left|\operatorname{Im}\left(\epsilon_{+}-\epsilon_{-}\right)\right| / t$ to characterize the PTsymmetry breaking transition. Here $e^{-i \epsilon_{ \pm} T / \hbar}$ are eigenvalues of the non-unitary time-evolution operator $U=\mathcal{T} e^{-i \int_{0}^{T} H(\tau) / \hbar d \tau}$, where $\mathcal{T}$ is the time-ordering operator. For $\lambda=0$, the system lies in the PTS phase, while $\lambda>0$ corresponds to a PTB phase.

Figure 1c shows a numerically calculated phase diagram with a fixed $\tau_{0} t$. The white PTS region is separated into several blocks (marked as $\mathcal{M}_{j}$ ), by a series of critical modulation frequencies $\Omega_{j}=$ $2 t / j\left(j \in \mathbb{N}^{+}\right)$at which the PTS phase vanishes and PT-symmetry breaking is at its maximum. The colored PTB regimes are further divided by the critical modulation frequencies into $\mathcal{L}_{j}$ and $\mathcal{V}_{j}$ regions, respectively, corresponding to quantum Zeno and antiZeno regimes, as we explicitly demonstrate later. For any fixed
$\Omega \neq \Omega_{j}$, a PTS to PTB transition $\left(\mathcal{M}_{j} \rightarrow \mathcal{L}_{j}\right.$ or $\left.\mathcal{M}_{j} \rightarrow \mathcal{V}_{j+1}\right)$ is crossed when increasing $\gamma_{0}$ from weak to strong. However, for a fixed $\gamma_{0}$, the PTS and PTB phases alternate $\left(\mathcal{M}_{j} \rightarrow \mathcal{L}_{j} \rightarrow \mathcal{V}_{j} \rightarrow \mathcal{M}_{j-1} \rightarrow\right.$ $\left.\mathcal{L}_{j-1} \rightarrow \cdots\right)$ with increasing modulation frequency $\Omega$. While the phase diagram is distinct from that of PT symmetric systems under a continuous dissipation ${ }^{29}$, a crucial observation is that quantum Zeno regimes exist in the PTB phases, and are bounded by critical frequencies, as well as by exceptional points pertaining to the PT symmetry breaking transitions.

\section{Experimental implementation}

To experimentally simulate the non-unitary dynamics driven by Hamiltonian (1), we embed the dissipative Hamiltonian (1) into a larger Hilbert space composed of atomic momentum states. As illustrated in Fig. 2a, a momentum lattice is generated by imposing multiple pairs of counter-propagating, far-detuned Bragg lasers (with the wavelength $\lambda_{0}=1064 \mathrm{~nm}$ ) on a Bose-Einstein condensate (BEC) of $\sim 10^{587} \mathrm{Rb}$ atoms in a weak 
a

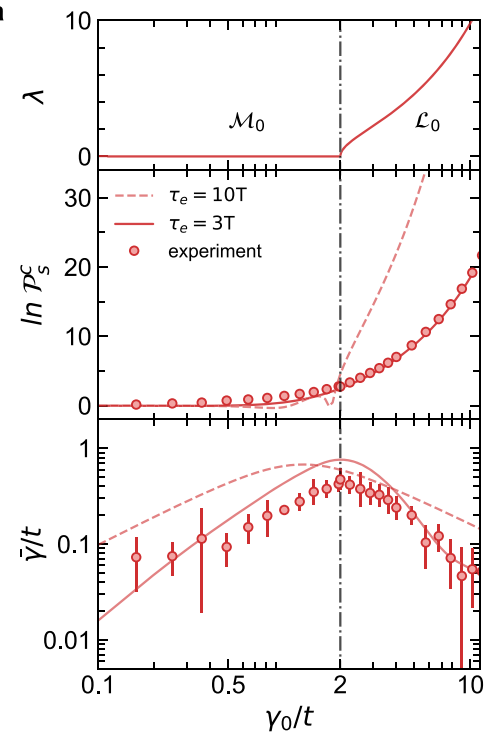

b

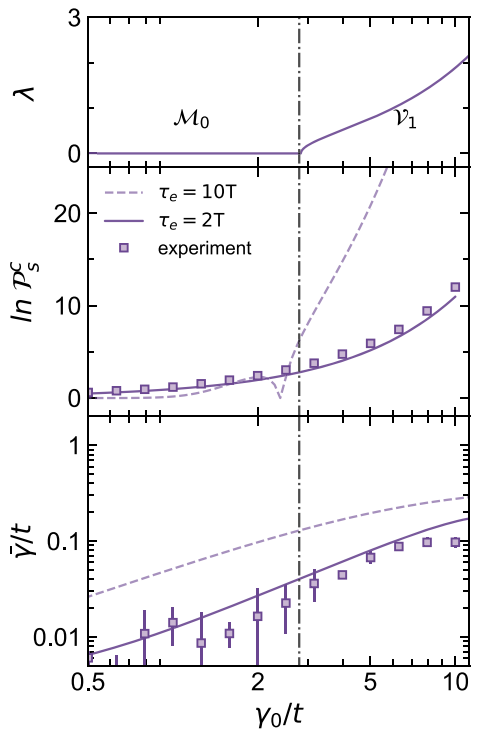

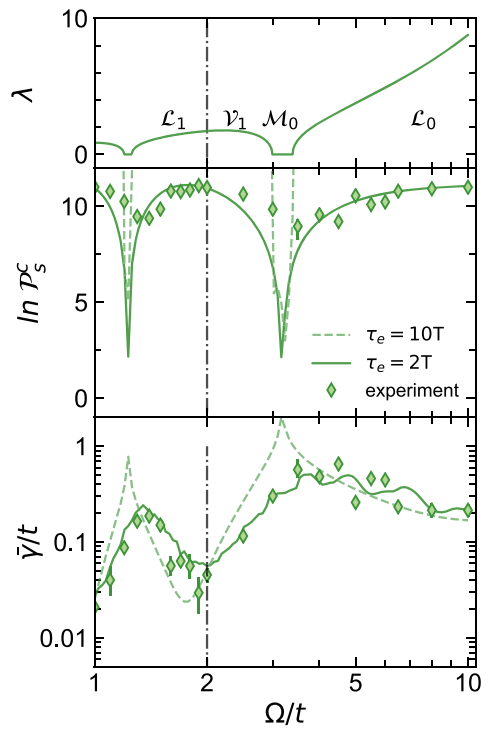

Fig. 3 Observation of the correspondence between quantum (anti-)Zeno effects and the PT phases. Here by varying the kick parameters, the PT phases and (anti-)Zeno effects are respectively indicated by numerically calculated dimensionless parameter $\lambda$ (upper panel), measured corrected Probability $\mathcal{P}_{s}^{c}$ (middle panel), and the measured effective loss rate $\bar{\gamma}$ (lower panel). a Dependence of $\lambda$, $\mathcal{P}_{s}^{c}$ and $\bar{\gamma}$ on the kick intensity $\gamma_{0}$ under a large kick frequency $\Omega / t=10$. The exceptional point is at $\gamma_{0} / t=2$ (dash-dotted line). b Dependence of $\lambda, \mathcal{P}_{s}^{c}$ and $\bar{\gamma}$ on during the duty time under a small kick frequency $\Omega / t=2.5$. The exceptional point is at $\gamma_{0} / t=2.8$ (dash-dotted line). c Dependence of $\lambda, \mathcal{P}_{s}^{c}$ and $\bar{\gamma}$ on the kick frequency $\Omega$ with a fixed $\gamma_{0} / t \sim 9$. The evolution $\tau_{e}=3 T$ for panel (a), while $\tau_{e}=2 T$ for panels (b) and (c). For all experiments, we take $t=$ $2 \pi \times 1.03(2) \mathrm{kHz}$, and the duty time is $\tau_{0} t /(2 \pi)=0.1$. In panels (a) and (b), we vary $\gamma_{0}$ by choosing different values of $t_{0}$. The solid (dashed) lines in the middle panels are numerical simulations with the experimentally applied $\tau_{e}$ (a longer $\tau_{\mathrm{e}}=10 T$ ), while the dashed (solid) lines in the lower panels are numerical simulations with Hamiltonian (1) (an effective Hamiltonian including higher-order, non-resonant coupling terms; see Methods). The vertical dash-dotted line in panel (c) indicates the critical kick frequency $\Omega_{1}=2 t$. All error bars here indicate one standard deviation from multiple measurements.

optical dipole trap ${ }^{33-35}$. The frequencies of the Bragg lasers are carefully designed to couple 8 discrete momentum states $p_{n}=2 n \hbar k\left(k=2 \pi / \lambda_{0}\right.$ and $\left.n=0,1, \ldots . .7\right)$, which form a synthetic lattice of finite size, with individually tunable Bragg-assisted tunneling strength $t_{n}$ between adjacent sites $|n-1\rangle$ and $|n\rangle$; see Fig. 2b, c. A unitary kick is then introduced through a square-wave modulation $t_{2}=t_{z}(\tau)$ for the inter-site coupling $|1\rangle \leftrightarrow|2\rangle$. Consistent with Eq. (2), $t_{z}(\tau)=t_{0}$ for $j T \leq \tau<j T+\tau_{0}$, while vanishes for other time intervals. Treating momentum-lattice sites $|n \geq 2\rangle$ as a reservoir (within which the coupling strength $t_{n>2}=t$ ), we find that dynamics within the two-dimensional subspace spanned by $\{|0\rangle,|1\rangle\}$ to be dissipative, and effectively driven by Hamiltonian (1) with $\gamma_{0} \sim t_{0}^{2} / t^{36}$. While the above expression of $\gamma_{0}$ is perturbatively valid for $t_{0} \ll t$, we find it capable of capturing the dissipative properties qualitatively well at short evolution times even for $t_{0} \sim t$. As such, we implement an effectively dissipative two-level system in momentum space, whose dissipation originates from unitary kicks that, with kick frequency $\Omega$ and intensity $\gamma_{0}$, periodically couple the system with a reservoir.

We study both the PT symmetry breaking transition and the quantum Zeno (anti-Zeno) effects through the dissipative dynamics. Specifically, we initialize the atoms in the state $|0\rangle$, and let them evolve for a short time $\tau_{e}$, before applying a time-offlight image to record the atomic probability distribution $P_{n}$ for each momentum lattice site, normalized by the total atom population over the momentum lattice (see Methods). Under the passive PT symmetric Hamiltonian (1), the PTS and PTB phases can be dynamically differentiated by the corrected probability ${ }^{31}$

$\mathcal{P}_{s}^{c}=\left(P_{0}+P_{1}\right) \exp \left(\gamma_{0} \tau_{0} \frac{\tau_{e}}{T}\right)$

which reflects the time evolution of the squared state norm within the synthetic subspace driven by the Hamiltonian $H_{P T}$. It follows that (see middle panels in Fig. 3), $\mathcal{P}_{s}^{c}$ should be on the order of unity in the PTS phase, while it should exponentially grow with time in the PTB phase. To further characterize quantum Zeno and anti-Zeno regimes, we probe the effective loss rate $\bar{\gamma}$ via

$e^{-\bar{\gamma} \tau_{e} / 2}=1-\mathcal{P}_{r}$,

where $\mathcal{P}_{r}$ is the total population loss of the dissipative two-level system during the time evolution up to $\tau_{e}$, with $\mathcal{P}_{r}=\sum_{n>2} P_{n}$. As we detail in the Supplementary Information, while quantum Zeno and anti-Zeno effects are typically defined as the change in decay of a given unstable state rather than that of the whole system, the effective loss rate $\bar{\gamma}$ extracted from Eq. (4) constitutes a reasonably good indicator of the quantum Zeno to anti-Zeno transition (as well as the PT phase transition), as long as $\tau_{e}$ is sufficiently long. While we typically fix $\tau_{e}$ to be two or three modulation periods, limited by both the finite size of the reservoir and the decoherence time of the system ${ }^{25,37}$, it is already long enough to reveal the transition point in our experiment.

\section{Quantum Zeno effect across PT phase transitions}

In Fig. 3a, we show the experimentally constructed corrected probability $\mathcal{P}_{s}^{c}$ and the effective loss rate $\bar{\gamma}$ across the PT phase transition $\mathcal{M}_{0} \rightarrow \mathcal{L}_{0}$ at a high kick frequency $\Omega / t=10$ and with increasing kick intensity $\gamma_{0}$. The corrected probability (middle panel) becomes exponentially large beyond the exceptional point at $\gamma_{0} / t \sim 2$ (dash-dotted vertical line from upper panel). In the PTS (PTB) phase $\mathcal{M}_{0}\left(\mathcal{L}_{0}\right)$, the effective loss rate of the synthetic twolevel system increases (decreases) with increasing $\gamma_{0}$ (see lower panel), indicating quantum anti-Zeno (Zeno) regime. The effective loss rate $\bar{\gamma}$ peaks near the exceptional point, consistent with the theoretical prediction that the quantum Zeno to anti-Zeno transition should coincide with the PTB-PTS transition. 
However, such is not the case at lower kick frequencies. As illustrated in Fig. 3b, when $\gamma_{0}$ is tuned at a fixed $\Omega / t=2.5, \bar{\gamma}$ increases monotonically across the transition $\mathcal{M}_{0} \rightarrow \mathcal{V}_{1}$ at $\gamma_{0} / t=2.8$ (dashdotted line), suggesting both the PT symmetric $\mathcal{M}_{0}$ and the PT broken $\mathcal{V}_{1}$ belong to the quantum anti-Zeno regime. Note that at the critical kick frequencies, for instance $\Omega_{1}=2 t, \bar{\gamma}$ also increases with increasing $\gamma_{0}$. Thus, quantum anti-Zeno effects survive at the boundaries between $\mathcal{V}_{j}$ and $\mathcal{L}_{j}$ in the PTB phase.

Apart from tuning $\gamma_{0}$, both the PT-symmetry breaking transition and quantum Zeno to anti-Zeno transition can be crossed by changing the kick frequency, which amounts to traversing the phase diagram Fig. 1 vertically. Figure $3 c$ shows the measured $\bar{\gamma}$ across multiple PT phase transitions by increasing $\Omega$ with a fixed $\gamma_{0} / t \sim 9$. The measured effective loss rate $\bar{\gamma}$ peaks near the PTsymmetry phase boundary between $\mathcal{M}_{j}$ and $\mathcal{L}_{j}$, consistent with the coincidence of the two transitions according to the theoretical phase diagram. Furthermore, a local minimum in $\bar{\gamma}$ is found near the critical kick frequency $\Omega_{1}=2 t$ (lower panel), where a "slow mode," i.e., the eigenstate with the smaller imaginary eigenvalue, dominates the dynamics ${ }^{32}$. We emphasize that the occurrence of quantum anti-Zeno effect in the PTB regime is unique to slow modulations. For fast modulations $(\Omega / t \gg 1$, where the transition $\mathcal{M}_{0} \rightarrow \mathcal{L}_{0}$ lies), increasing the kick rate is similar to enlarging the dissipation rate in the continuous case ${ }^{23,32}$. There, only a single transition point from the quantum anti-Zeno to Zeno regime exists, whic $\hbar \mathrm{h}$ occurs exactly at the exceptional point.

Experimental measurements in Fig. 3 qualitatively agree with theoretical predictions, since both the weak-coupling (i.e., the coupling strength $\hbar t_{n} \ll 8 E_{r}$ with $\left.E_{r}=\hbar^{2} k^{2} / 2 m\right)$ and weakinteraction (the interaction strength much smaller compared with the $\hbar t_{n}$ ) conditions are satisfied throughout our experiments. Nevertheless, quantitative deviations exist, which mainly derive from two sources. First, the kick intensity $\gamma_{0}$ in the effective Hamiltonian (1) would deviate from the perturbative expression $\gamma_{0} \sim t_{0}^{2} / t$ when either the coupling $t_{0}$ or the evolution time becomes sufficiently large. This is the main reason for the slight discrepancy between the location of the maximum loss rate in Fig. 3a, either numerically simulated (dashed and solid lines) or experimentally measured, and that of the theoretically predicted exceptional point using the perturbative kick intensity (dashdotted). Second, high-order, non-resonant coupling terms play an important role in our experiment, as is manifest in Fig. 3 where the experimental data agree better with simulations considering the non-resonant coupling terms (solid lines). As non-resonant couplings enable the $|0\rangle \leftrightarrow|-1\rangle$ transition, the population of the $|-1\rangle$ state leads to an underestimation of loss for a finite evolution time. Other factors, for example, interaction-induced self-trapping in the momentum lattice and the momentum broadening due to the weak trap potential ${ }^{25,37,38}$, also lead to underestimations of the loss rate. These experimental imperfections lead to an overestimation of the corrected probability $\mathcal{P}_{s}^{c}$ (middle panels in Fig. 3), while the overall measured profiles still qualitatively agree with the theoretical predictions on the PT phase transition.

\section{Correspondence between quantum (anti-)Zeno effects and PT phases}

Finally, we map out the phase diagram for quantum Zeno to antiZeno transition by sweeping $t_{0}$ (hence $\gamma_{0}$ ) for a set of fixed $\Omega$, and plotting the quantity $\kappa \bar{\gamma}$ with $\kappa=\operatorname{sgn}\left(\Delta \bar{\gamma} / \Delta \gamma_{0}\right)$; see Fig. 4. Here the difference $\Delta \bar{\gamma} / \Delta \gamma_{0}$ is calculated from experimental data for each fixed $\Omega$. By definition, $k \bar{\gamma}<0(\kappa \bar{\gamma}>0)$ represents the quantum Zeno (anti-Zeno) regime. At the lower-right corner of Fig. $4, k \bar{\gamma}$ is close to zero, due to a vanishing $t_{z}$ and a disconnected reservoir. At the upper-left corner, $k \bar{\gamma}$ also approaches zero, as loss to the reservoir is suppressed, which is equivalent to the standard quantum Zeno effect in the case of continuous, strong couplings.

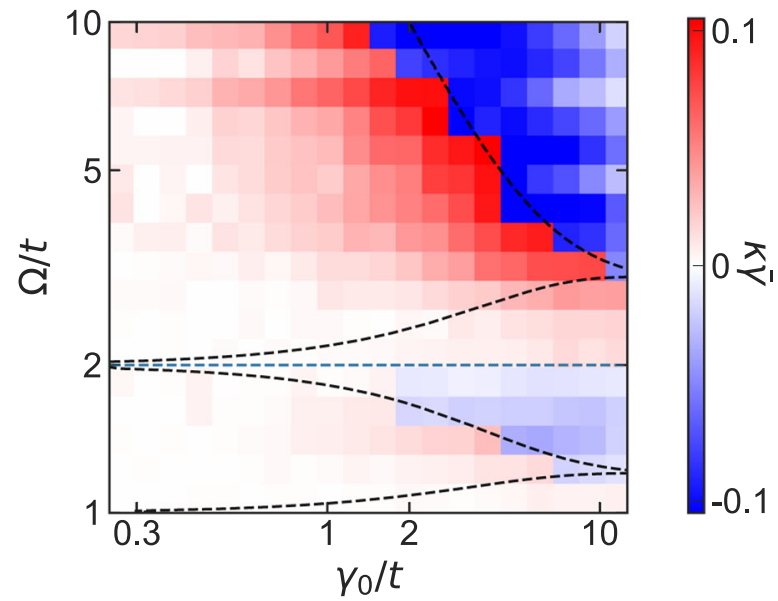

Fig. 4 Phase diagram for quantum anti-Zeno to Zeno transition from experimental data. Color contour is the measured $k \bar{\gamma}$ (see main text for definition). The black dashed lines indicate the exceptional points [see Fig. 1(c)], and the blue dashed line corresponds to the critical kick frequency $\Omega_{1}=2 t$. The blue (red) regions correspond to quantum Zeno (anti-Zeno) regimes. All $\bar{\gamma}$ are measured after the system evolves for two modulation periods, while we set $t=2 \pi \times 1.03(2) \mathrm{kHz}$ and $\tau_{0} t /(2 \pi)=0.1$ for all measurements. The measured critical anti-Zeno to Zeno transition points are consistent with the exceptional points between the $\mathcal{M}_{j}$ and $\mathcal{L}_{j}$ regions.

Most importantly, by superimposing the boundaries of PT transitions (black dashed) and the critical kick frequency (blue dashed), it is clear that our measured phase diagram in Fig. 4 agrees well with the theoretical prediction in Fig. 1c, thus confirming the following correspondence

$$
\begin{aligned}
\mathcal{V}_{j}(\mathrm{PTB}), \mathcal{M}_{j}(\mathrm{PTS}) & \leftrightarrow \text { anti-Zeno } \\
\mathcal{L}_{j}(\mathrm{PTB}) & \leftrightarrow \text { Zeno } .
\end{aligned}
$$

Such a relationship reveals the deep connection between PT transition and quantum Zeno effects.

However, we note that both quantum Zeno and anti-Zeno effects can occur in dissipative systems without PT symmetry and devoid of exceptional points ${ }^{39,40}$. For instance, by considering a system with an additional diagonal detuning $\delta \sigma_{z}$ ( $\delta$ being real $)^{41,42}$, slow-decaying modes emerge that give rise to antiZeno effects ${ }^{40}$, even in the absence of PT symmetry. Therefore, the elegant correspondence in Eq. (5) should be understood in the context of PT symmetric systems.

\section{DISCUSSION}

To conclude, we have experimentally established the connection between the quantum Zeno effect and PT phases in a dissipative Floquet system: while the PTS phase generally leads to the quantum anti-Zeno effect, both quantum Zeno and anti-Zeno effects can occur in the PTB region. Crucially, the quantum-Zeno regimes are bounded by a discrete set of critical coupling frequencies, and by exceptional points. Besides shedding new lights on the relation of quantum measurements and dynamics of non-Hermitian systems, our experiment also offers a new way of simulating PT physics using cold atoms, which is readily extendable to higher dimensions (see Supplementary Information). While quantum Zeno effects and the associated quantum Zeno subspace $^{23}$ generally exist for multi-level systems, the scalability of the correspondence considered here to higher dimensions is an interesting open question that we leave to future studies. 
Furthermore, the above analyses are all within the scope of single-particle physics, without considering the effect of interactions. Specifically, many-body interactions in the momentum lattice assume the form of density-dependent, attractive on-site potentials ${ }^{38}$. When the atomic density or the scattering length is large enough, atoms in momentum space exhibit the so-called interaction-induced localization ${ }^{37,38}$. Since the quantum Zeno dynamics can also be regarded as a form of localization (or stabilization) within the quantum Zeno subspace ${ }^{43-45}$, it will be interesting to study the interplay between interactions and quantum Zeno effects in future experiments ${ }^{46-49}$.

\section{METHODS}

\section{Experimental settings}

The ${ }^{87} \mathrm{Rb}$ BEC is prepared in an optical dipole trap. The multiple discrete momentum states are coupled with multi-frequency Bragg laser pairs. The different frequency components are imprinted by two acoustic optical modulators. One shifts the frequency of the incoming beam by $-100 \mathrm{MHz}$, and another shifts it by $100 \mathrm{MHz}-\Sigma_{n} v_{n} / 2 \pi(n \geq 1)$ with $v_{n}=4(2 n-1) \hbar k^{2} /$ $2 m$ (see the main text). As a consequence, the transition between the two momentum states, $|n-1\rangle \leftrightarrow|n\rangle$, can be resonantly triggered by the $\left\{\omega_{+}\right.$, $\left.\omega_{n}\right\}$ laser pair.

After the system evolves for a finite time $\tau_{e}$, we directly resolve the populations in each momentum state by letting the atoms fall freely in space for $20 \mathrm{~ms}$ with all lasers switched off, before the atoms are imaged by a camera. Atoms with different momenta get separated in the $x$ direction along which the Bragg beams are applied (see Fig. 1 in the main text). To obtain the relative populations in each state, we integrate the image in the $y$-direction, and then fit the data with a 10-peak Gauss function, $\mathcal{A}(x)=\sum_{n=-1}^{8} A_{n} \exp \left[-\left(\frac{x-n d}{a}\right)^{2}\right]$. Normalizing the resulting amplitude $\mathcal{A}_{n}$ by $\sum_{n=-1}^{8} \mathcal{A}_{n}$, we finally get the atom population distribution on each site, $P_{n}$.

\section{Effective Hamiltonian with off-resonant terms}

Following the theory of light-atom interaction in ref. ${ }^{33}$, we obtain the effective time-dependent full Hamiltonian

$$
H_{\text {eff }}=\sum_{n} \sum_{i} \hbar \frac{\Omega_{+} \Omega_{i}}{4|\Delta|} e^{i\left[\left(\omega_{+}-\omega_{i}-4(2 n-1) \hbar k^{2} / 2 m\right) t+\left(\phi_{+}-\phi_{i}\right)\right]}|n-1\rangle\langle n|
$$

with $\phi_{+}$and $\phi_{i}$ the phases of beams with frequencies $\omega_{+}$and $\omega_{i r}$ respectively (see Fig. 2 in the main text). We simply let $\phi_{+}=0$, and $\phi_{i}$ be the modulated phase relative to $\phi_{+}$from the AOM. As we choose $\omega_{i}=\omega_{+}$ $-4(2 i-1) \hbar k^{2} / 2 m$, the simplified ideal model can be obtained by considering only the resonant terms, i.e., letting $i=n$, as

$$
H^{(0)}=\sum_{n} \hbar t_{n}|n-1\rangle\langle n|+\text { H.c. }
$$

with $t_{n}=e^{-i \phi_{n}} \Omega_{+} \Omega_{n} / 4|\Delta|$. This gives the general tight-binding form for a momentum-state chain. If we simply treat the $n \geq 2$ part as an effective reservoir, and apply the second-order perturbation with $t_{2}=t_{z}$ and $t_{n \neq 2}=t_{\text {, }}$ the loss rate of site $|1\rangle$ should approximately be $\gamma_{0} \sim t_{z}^{2} / t^{36}$. Then we obtain the dissipative two-level Hamiltonian in the main text.

Clearly, the $\ell$ th-order non-resonant terms, responsible for the transition $|n-1\rangle \leftrightarrow|n\rangle$, can be induced by $\left\{\omega_{+}, \omega_{n-\ell}\right\}$ and $\left\{\omega_{+}, \omega_{n+\ell}\right\}$ laser pairs with detunings of $\mp 8 \ell \hbar k^{2} / 2 m$, respectively. These terms are given by

$$
H^{(\ell)}=\sum_{n} \hbar t_{n \pm \ell} e^{ \pm i 8 \ell\left(\hbar k^{2} / 2 m\right) t}|n-1\rangle\langle n|+\text { H.C. },
$$

leading the full Hamiltonian $H_{\text {eff }}=\sum_{e} H^{(\ell)}$. In our experiment, $8 \hbar k^{2} / 2 m$ corresponds to $\sim 2 \pi \times 16.2 \mathrm{kHz}$.

\section{DATA AVAILABILITY}

The datasets generated during and/or analyzed during the current study are available from the corresponding author on reasonable request.

Received: 11 November 2020; Accepted: 17 April 2021; Published online: 24 May 2021

\section{REFERENCES}

1. Itano, W. M., Heinzen, D. J., Bollinger, J. J. \& Wineland, D. J. Quantum Zeno effect. Phys. Rev. A 41, 2295-2300 (1990).

2. Fischer, M. C., Gutiérrez-Medina, B. \& Raizen, M. G. Observation of the quantum Zeno and anti-Zeno effects in an unstable system. Phys. Rev. Lett. 87, 040402 (2001)

3. Streed, E. W. et al. Continuous and pulsed quantum Zeno effect. Phys. Rev. Lett. 97, 260402 (2006).

4. Kilina, S. V., Neukirch, A. J., Habenicht, B. F., Kilin, D. S. \& Prezhdo, O. V. Quantum Zeno effect rationalizes the phonon bottleneck in semiconductor quantum dots. Phys. Rev. Lett. 110, 180404 (2013).

5. Harrington, P. M., Monroe, J. T. \& Murch, K. W. Quantum Zeno effects from measurement controlled qubit-bath interactions. Phys. Rev. Lett. 118, 240401 (2017).

6. Wang, X.-B., You, J. Q. \& Nori, F. Quantum entanglement via two-qubit quantum Zeno dynamics. Phys. Rev. A 77, 062339 (2008).

7. Maniscalco, S., Francica, F., Zaffino, R. L., Lo Gullo, N. \& Plastina, F. Protecting entanglement via the quantum Zeno effect. Phys. Rev. Lett. 100, 090503 (2008).

8. Shao, X.-Q., Chen, L., Zhang, S. \& Yeon, K.-H. Fast CNOT gate via quantum Zeno dynamics. J. Phys. B 42, 165507 (2009).

9. Chandrashekar, C. M. Zeno subspace in quantum-walk dynamics. Phys. Rev. A 82 052108 (2010).

10. de Lange, G., Wang, Z. H., Ristè, D., Dobrovitski, V. V. \& Hanson, R. Universal dynamical decoupling of a single solid-state spin from a spin bath. Science 330, 60-63 (2010).

11. Smerzi, A. Zeno dynamics, indistinguishability of state, and entanglement. Phys. Rev. Lett. 109, 150410 (2012).

12. Paz-Silva, G. A., Rezakhani, A. T., Dominy, J. M. \& Lidar, D. A. Zeno effect for quantum computation and control. Phys. Rev. Lett. 108, 080501 (2012).

13. Zhu, B. et al. Suppressing the loss of ultracold molecules via the continuous quantum Zeno effect. Phys. Rev. Lett. 112, 070404 (2014).

14. Kalb, N. et al. Experimental creation of quantum Zeno subspaces by repeated multi-spin projections in diamond. Nat. Commun. 7, 13111 (2016).

15. Hacohen-Gourgy, S., García-Pintos, L. P., Martin, L. S., Dressel, J. \& Siddiqi, I. Incoherent qubit control using the quantum Zeno effect. Phys. Rev. Lett. 120, 020505 (2018).

16. Szombati, D. et al. Quantum rifling: protecting a qubit from measurement back action. Phys. Rev. Lett. 124, 070401 (2020).

17. Schäfer, F. et al. Experimental realization of quantum Zeno dynamics. Nat. Commun. 5, 3194 (2014).

18. Signoles, A. et al. Confined quantum Zeno dynamics of a watched atomic arrow. Nat. Phys. 10, 715-719 (2014).

19. Bretheau, L., Campagne-lbarcq, P., Flurin, E., Mallet, F. \& Huard, B. Quantum dynamics of an electromagnetic mode that cannot contain $\mathrm{n}$ photons. Science 348, 776-779 (2015)

20. Barontini, G., Hohmann, L., Haas, F., Estève, J. \& Reichel, J. Deterministic generation of multiparticle entanglement by quantum Zeno dynamics. Science $\mathbf{3 4 9}$ 1317-1321 (2015).

21. Do, H. V., Gessner, M., Cataliotti, F. S. \& Smerzi, A. Measuring geometric phases with a dynamical quantum Zeno effect in a Bose-Einstein condensate. Phys. Rev. Res. 1, 033028 (2019).

22. Kofman, A. G. \& Kurizki, G. Acceleration of quantum decay processes by frequent observations. Nature 405, 546 (2000).

23. Facchi, P. \& Pascazio, S. Quantum Zeno dynamics: mathematical and physical aspects. J. Phys. A 41, 493001 (2008).

24. Zhou, L., Yi, W. \& Cui, X. Dissipation-facilitated molecules in a fermi gas with nonhermitian spin-orbit coupling. Phys. Rev. A 102, 043310 (2020).

25. Gou, W. et al. Tunable nonreciprocal quantum transport through a dissipative aharonov-bohm ring in ultracold atoms. Phys. Rev. Lett. 124, 070402 (2020).

26. Bender, C. M. \& Boettcher, S. Real spectra in non-hermitian hamiltonians having pt symmetry. Phys. Rev. Lett. 80, 5243-5246 (1998).

27. Konotop, V. V., Yang, J. \& Zezyulin, D. A. Nonlinear waves in $\mathcal{P} \mathcal{T}$-symmetric systems. Rev. Mod. Phys. 88, 035002 (2016).

28. El-Ganainy, R. et al. Non-hermitian physics and $\mathcal{P} \mathcal{T}$ symmetry. Nat. Phys. 14, 11 (2018).

29. Naghiloo, M., Abbasi, M., Joglekar, Y. N. \& Murch, K. W. Quantum state tomography across the exceptional point in a single dissipative qubit. Nat. Phys. 15, 1232 (2019).

30. Wu, Y. et al. Observation of parity-time symmetry breaking in a single-spin system. Science 364, 878-880 (2019)

31. Li, J. et al. Observation of parity-time symmetry breaking transitions in a dissipative floquet system of ultracold atoms. Nat. Commun. 10, 855 (2019).

32. Li, J., Wang, T., Luo, L., Vemuri, S. \& Joglekar, Y. N. Unification of quantum Zeno-anti Zeno effects and parity-time symmetry breaking transitions. arXiv 2004.01364 (2020) 
33. Gadway, B. Atom-optics approach to studying transport phenomena. Phys. Rev. A 92, 043606 (2015)

34. Meier, E. J., An, F. A. \& Gadway, B. Atom-optics simulator of lattice transport phenomena. Phys. Rev. A 93, 051602 (2016).

35. Xie, D., Gou, W., Xiao, T., Gadway, B. \& Yan, B. Topological characterizations of an extended su-schrieffer-heeger model. npj Quantum Inf. 5, 55 (2019).

36. Lapp, S., Ang'ong'a, J., An, F. A. \& Gadway, B. Engineering tunable local loss in a synthetic lattice of momentum states. N. J. Phys. 21, 045006 (2019).

37. Xie, D. et al. Topological quantum walks in momentum space with a BoseEinstein condensate. Phys. Rev. Lett. 124, 050502 (2020).

38. An, F. A., Meier, E. J., Ang'ong'a, J. \& Gadway, B. Correlated dynamics in a synthetic lattice of momentum states. Phys. Rev. Lett. 120, 040407 (2018).

39. de J. Leon-Montiel, R. et al. Observation of slowly decaying eigenmodes without exceptional points in floquet dissipative synthetic circuits. Commun. Phys. 1, 88 (2018).

40. Joglekar, Y. N. \& Harter, A. K. Passive parity-time-symmetry-breaking transitions without exceptional points in dissipative photonic systems. Photon. Res. 6, A51 (2018).

41. Duan, L., Wang, Y.-Z. \& Chen, Q.-H. P T symmetry of a square-wave modulated two-level system. Chin. Phys. Lett. 37, 081101 (2020).

42. Harter, A. K. \& Joglekar, Y. N. Connecting active and passive $\mathcal{P} \mathcal{T}$-symmetric Floquet modulation models. Prog. Theor. Exp. Phys. 2020, 12 A106 (2020).

43. Barontini, G. et al. Controlling the dynamics of an open many-body quantum system with localized dissipation. Phys. Rev. Lett. 110, 035302 (2013).

44. Patil, Y. S., Chakram, S. \& Vengalattore, M. Measurement-induced localization of an ultracold lattice gas. Phys. Rev. Lett. 115, 140402 (2015).

45. Peise, J. et al. Interaction-free measurements by quantum Zeno stabilization of ultracold atoms. Nat. Commun. 6, 6811 (2015).

46. Everest, B., Lesanovsky, I., Garrahan, J. P. \& Levi, E. Role of interactions in a dissipative many-body localized system. Phys. Rev. B 95, 024310 (2017).

47. Rubio-Abadal, A. et al. Many-body delocalization in the presence of a quantum bath. Phys. Rev. X 9, 041014 (2019).

48. Bouganne, R., Aguilera, M. B., Ghermaoui, A., Beugnon, J. \& Gerbier, F. Anomalous decay of coherence in a dissipative many-body system. Nat. Phys. 16, 21 (2020).

49. Choi, J. et al. Robust dynamic hamiltonian engineering of many-body spin systems. Phys. Rev. X 10, 031002 (2020).

\section{ACKNOWLEDGEMENTS}

We acknowledge support from the National Key R\&D Program of China under Grant Nos. 2018YFA0307200, 2016YFA0301700, and 2017YFA0304100, the National Natural Science Foundation of China under Grant Nos. 12074337, 11974311, and 91736209, the Natural Science Foundation of Zhejiang province under Grant Nos. LR21A040002 and LZ18A040001, Zhejiang Province Plan for Science and Technology No. 2020C01019, and the Fundamental Research Funds for the Central Universities.

\section{AUTHOR CONTRIBUTIONS}

The experimental work and data analysis were carried out by T.C., W.G., D.X., T.X., and B.Y. Theoretical modeling and calculations were done by T.C., W.Y., and J.J. All authors discussed the results and contributed to the preparation of the manuscript.

\section{COMPETING INTERESTS}

The authors declare no competing interests.

\section{ADDITIONAL INFORMATION}

Supplementary information The online version contains supplementary material available at https://doi.org/10.1038/s41534-021-00417-y.

Correspondence and requests for materials should be addressed to J.J. or B.Y.

Reprints and permission information is available at http://www.nature.com/ reprints

Publisher's note Springer Nature remains neutral with regard to jurisdictional claims in published maps and institutional affiliations.

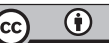

Open Access This article is licensed under a Creative Commons Attribution 4.0 International License, which permits use, sharing, adaptation, distribution and reproduction in any medium or format, as long as you give appropriate credit to the original author(s) and the source, provide a link to the Creative Commons license, and indicate if changes were made. The images or other third party material in this article are included in the article's Creative Commons license, unless indicated otherwise in a credit line to the material. If material is not included in the article's Creative Commons license and your intended use is not permitted by statutory regulation or exceeds the permitted use, you will need to obtain permission directly from the copyright holder. To view a copy of this license, visit http://creativecommons. org/licenses/by/4.0/.

(c) The Author(s) 2021 